\title{
DNA repair mechanisms and gametogenesis
}

\author{
Willy M. Baarends ${ }^{1}$, Roald van der Laan ${ }^{2}$ \\ and J. Anton Grootegoed ${ }^{1}$ \\ ${ }^{1}$ Department of Endocrinology and Reproduction and ${ }^{2}$ MGC Department of Cell \\ Biology and Genetics (Centre for Biomedical Genetics), Erasmus University Rotterdam, \\ PO Box 1738, 3000 DR Rotterdam, The Netherlands
}

\begin{abstract}
In mammals, there is a complex and intriguing relationship between DNA repair and gametogenesis. DNA repair mechanisms are involved not only in the repair of different types of DNA damage in developing germline cells, but also take part in the meiotic recombination process. Furthermore, the DNA repair mechanisms should tolerate mutations occurring during gametogenesis, to a limited extent. In the present review, several gametogenic aspects of DNA mismatch repair, homologous recombination repair and postreplication repair are discussed. In addition, the role of DNA damage-induced cell cycle checkpoint control is considered briefly. It appears that many genes encoding proteins that take part in DNA repair mechanisms show enhanced or specialized expression during mammalian gametogenesis, and several gene knockout mouse models show male or female infertility. On the basis of such knowledge and models, future experiments may provide more information about the precise relationship between DNA repair, chromatin dynamics, and genomic stability versus instability during gametogenesis.
\end{abstract}

The stability of genetic information is of vital importance for normal function and reproduction of all living organisms. In heterogametic species, this stability should be maintained in somatic cells, but also and in particular in the germline cells. The male and female gametes are the basis of a new individual, and their intact genomes ensure faithful transmission of genetic information to the next generation. However, evolution would not be possible if the genomes of the gametes did not contain some mutations. Hence, gametogenesis should tolerate a limited number of mutations. These two contradictory requirements of genome stability versus instability must be achieved on the basis of DNA repair pathways, which are active in virtually all somatic cell types and may show specific properties and activities during gametogenesis (Fig. 1).

Bacteria and eukaryotic species from yeast to mammals have an impressive number of highly interactive multienzyme mechanisms targeting DNA, catalysing and controlling DNA replication, transcription and repair. The mechanisms functioning primarily in DNA repair include nucleotide excision repair, base excision repair, mismatch repair, homologous recombination repair and post-replication repair. This review will focus on mismatch repair, homologous recombination repair and post-replication repair in the context of mammalian gametogenesis. In addition, some

Email: baarends@endov.fgg.eur.nl attention is given to DNA damage-induced cell cycle checkpoint control.

\section{Mismatch repair}

In copying the total genome before cell division, the DNA replication machinery makes mistakes, approximately one error per $10^{7}$ incorporated nucleotides. Most of these mistakes are corrected by DNA mismatch repair (Fig. 2). Many different proteins are involved in DNA mismatch repair, and the names given to these proteins in eukaryotic species are based on their homology with proteins involved in mismatch repair in prokaryotes, MutS and MutL, and the yeast protein PMS1, which is involved in mismatch repair and also in post-meiotic segregation (hence, the abbreviation PMS). In spite of its different name, PMS1 is a homolog of MutL (in the field of DNA repair, much attention is required to understand the names and abbreviations, which can confuse even insiders). In humans and mice, there are many MutS and MutL homologues, named MSH1-6, MLH1 and MLH3, and PMS1 and PMS2 (Arnheim and Shibata, 1997; Lipkin et al., 2000).

A heterodimer of two different MutL proteins is thought to interact with a heterodimer of two different MutS proteins. Such a heterotetrameric complex can recognize a single base-pair mismatch, or a small loop of single-stranded DNA, depending on the composition of the complex. Endo-exonucleases and other proteins involved in DNA 


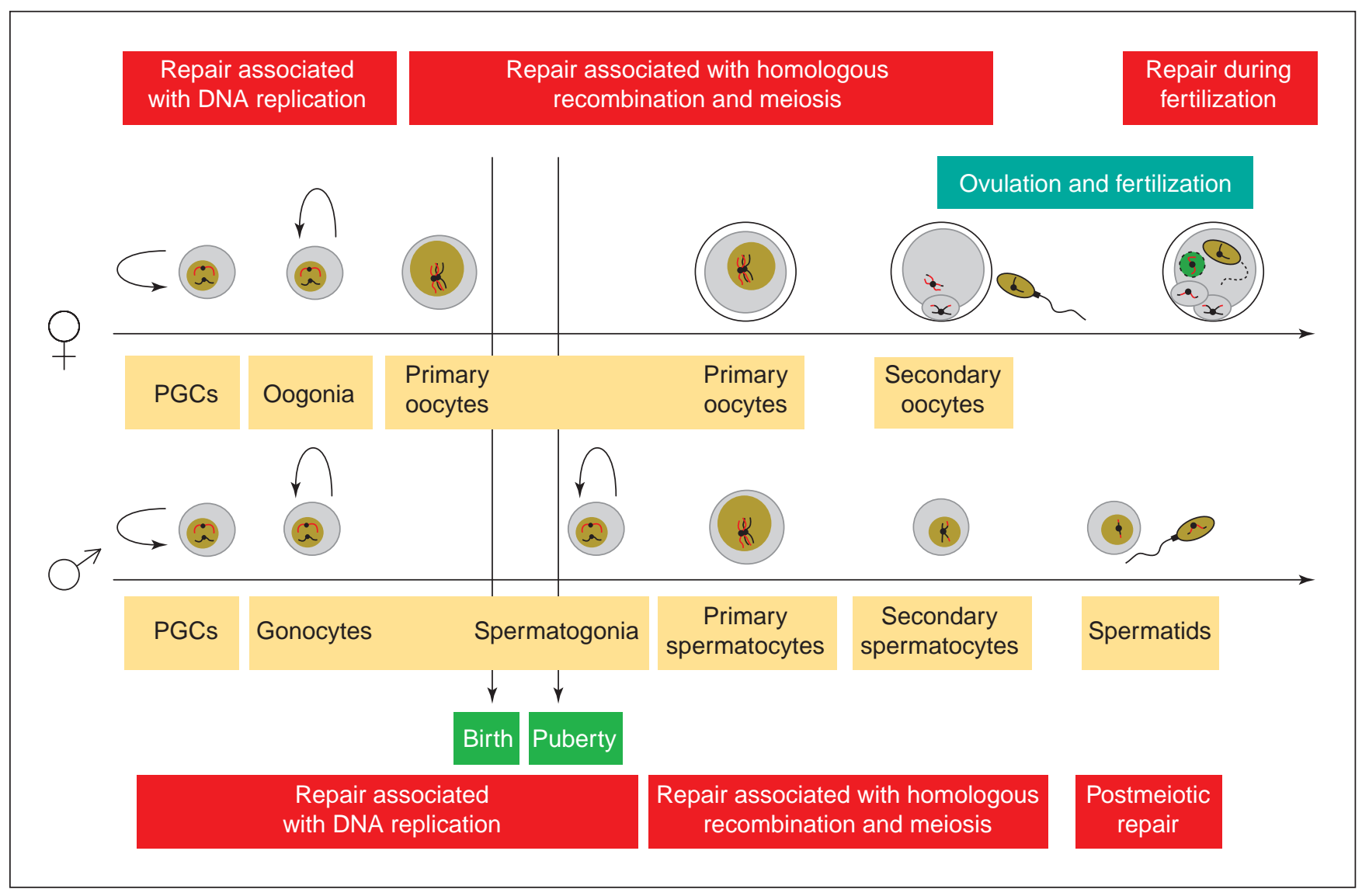

Fig. 1. Mammalian gametogenesis and DNA repair mechanisms. During mitotic, meiotic and postmeiotic development of male and female germ cells, and during fertilization, specific DNA repair mechanisms show different activities. The developmental phases of male (lower panel) and female (upper panel) gametogenesis are shown in relation to DNA repair activities. Note that DNA repair associated with mitotic DNA replication takes place throughout life in males, whereas, in females, it operates only during fetal development. The lifespan of meiotic cells in females may be years or decades, whereas, in males, meiosis takes place in a fixed time schedule of about 2 weeks. PGCs: primordial germ cells.

replication take part in the mismatch repair DNA-protein complex, to bring about repair of the mismatch (Arnheim and Shibata, 1997).

Mismatch repair would be expected to be essential for the prevention of gene mutations during mitotic expansion and maintenance of the germline. Approximately 20 mitotic divisions precede the formation of primary oocytes during human fetal development. The male premeiotic germ cells may have gone through more than 1000 mitotic divisions before the spermatogonia enter the meiotic prophase in a 50-year-old male (Hurst and Ellegren, 1998). The very high number of mitotic divisions in the male germline has led to suggestions that male gametogenesis rather than female gametogenesis drives evolution (Agulnik et al., 1997). An estimated $0.1 \%$ of mutations produced by the DNA replication machinery escape mismatch repair, and accumulation of several mutations in the male germline is to be expected when these mutations do not lead to germ cell death.

Information about the roles of the various components of the mismatch repair system in gametogenesis has been gained in recent years from analysis of mouse gene knockout models, containing a homozygous null mutation of a gene encoding one of these components. Knockout mice for one of the genes MSH2, MSH3 and MSH6 show pronounced mismatch repair deficiency for somatic cells, but fertility appears to be normal (Arnheim and Shibata, 1997; de Wind et al., 1999). However, several other MLH-MSH-PMS homologues appear to be involved in gametogenesis, with or without an additional role in mismatch repair in somatic cells.

Targeted mutation of the $\mathrm{MLH1}$ gene results in a phenotype showing genomic instability, as demonstrated by changes in the length of dinucleotide repeats, and a predisposition to certain tumours (Baker et al., 1996; Edelmann et al., 1996). Human germline mutations in MLH1 cause hereditary nonpolyposis colon cancer (Arnheim and Shibata, 1997). The phenotype of MLH1 knockout mice also includes male and female infertility, owing to pachytene arrest (Baker et al., 1996; Edelmann et al., 
1996). Meiotic recombination is severely impaired in MLH1 knockout mice, which display univalency of chromosomes. The MLH1 protein has been localized to 'early-' and 'laterecombination' nodules on the synaptonemal complex (a proteinaceous axis formed along the length of the paired chromosomes during meiotic prophase) of pachytene spermatocytes and oocytes (Baker et al., 1996). The average number of MLH1 foci detected in mid-to-late pachytene cells using an anti-MLH1 antibody corresponds to the known average number of cross-overs (which differs between oocytes and spermatocytes, and among species). These observations, and additional data from yeast mutant models (Wang et al., 1999) are in agreement with a role for MLH1 in promoting crossing-over.

Similar to the MLH1 knockout mice, PMS2 knockout mice have a phenotype associated with overall genomic instability. However, with respect to changes in fertility, the PMS2 knockout mice develop male-restricted infertility (Baker et al., 1995). In the spermatocytes of these mice, pairing of homologous chromosomes is impaired, but there is no complete meiotic arrest and some malformed spermatids are still produced. Baker et al. (1995) suggested that the PMS2 protein is involved in promoting the pairing of homologous chromosomes, and in the stimulation of the formation of early recombination intermediates. This action of PMS2 would be expected to impair the meiotic prophase in fetal oocytes, but for reasons that are not well understood, the oocytes can also complete meiotic chromosome pairing in the absence of PMS2.

In addition to MLH1 and PMS2, there are two other MutL homologues in mice and humans: PMS1, which is most likely involved in the repair of mitochondrial DNA mismatches; and MLH3. Meiosis-specific MutL homologues have not been identified. However, there are two mammalian meiosis-specific MutS homologues, MSH4 and MSH5 (Paquis-Flucklinger et al., 1997; Winand et al., 1998). Targeted inactivation of either one of these genes results in a gametogenesis-specific phenotype in both cases, displaying male and female meiotic arrest (de Vries et al., 1999; Edelmann et al., 1999; Kneitz et al., 2000). In the MSH4 and MSH5 knockout mice, meiosis proceeds up to the zygotene stage, and pairing of homologous chromosomes is greatly diminished. This decline in chromosome pairing is most severe in the MSH5 knockout. Certain functions of these proteins are most likely performed in heteromeric complexes also containing the MutL homologues, MLH1 or PMS2. In support of this concept, human MSH4 has been shown to interact with MLH1, and the two proteins partially colocalize on the synaptonemal complex during spermatogenesis in mice (Kneitz et al., 2000; Santucci-Darmanin et al., 2000).

These studies have emphasized that mismatch repair plays a role not only in mitotic proliferation, but also in meiosis. During proper homologous recombination, a single DNA strand of one chromatid undergoes pairing with one of the two strands of a non-sister chromatid, and this paired stretch may contain several mismatches that can be

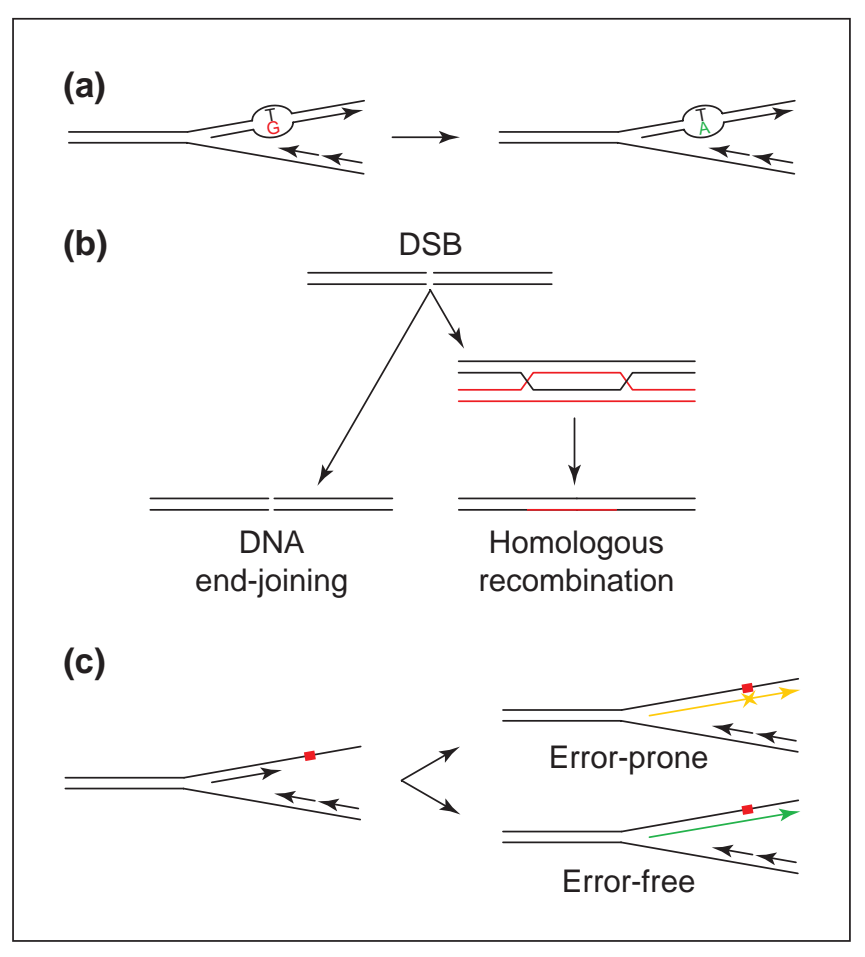

Fig. 2. Schematic and highly simplified representation of three DNA repair mechanisms. (a) Mismatch repair: around the mismatch, a gap is formed and then filled in by repair synthesis, followed by ligation of the ends. (b) Double strand break (DSB) repair. DNA-ends are joined and ligated (error-prone), or the DSB is repaired by homologous recombination, where homologous strands interact after branch migration, followed by repair synthesis, resolution of cross-overs, and ligation (error-free). (c) Postreplication repair. Specialized polymerases allow replication over a damaged template in an error-prone or an error-free manner; the error-prone mechanism often results in a mutation.

recognized and repaired by the mismatch repair system. Furthermore, although there is precise alignment of the homologous chromosomes during the early meiotic prophase, it remains important to suppress recombination between mismatched repeated sequences, which are located at many sites in the genome. The mismatch repair system appears to be active in suppression of such unwarranted heteroduplexes.

In conclusion, the mismatch repair pathway may repair mismatches occurring during the many rounds of mitotic DNA replication that precede meiosis, in particular in males. However, a major role of the mismatch repair pathway in gametogenesis appears to involve control of several aspects of meiotic chromosome pairing and recombination. This second role is emphasized by the functional importance of gametogenesis-specific mammalian MSH proteins.

\section{Homologous recombination repair}

Breakage of DNA in living cells that results in a DNA double strand break (DSB) is a major accident. However, 


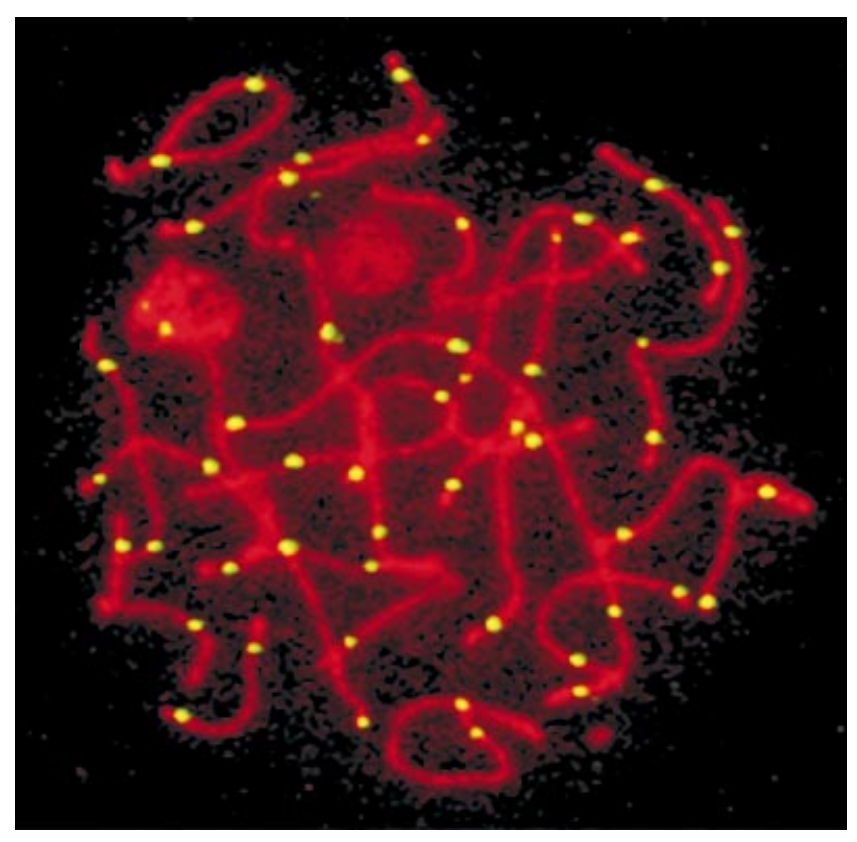

Fig. 3. MLH1 foci representing cross-over sites in a human spermatocyte nucleus. The proteinaceous axes (synaptonemal complexes) between the paired chromosomes of this mid-pachytene human spermatocyte are immunostained with anti-SCP3 (red). The MLH1 foci (green) localize on the axes.

DSBs often can be repaired effectively by a relatively simple mechanism, which involves DNA-end-joining by ligation of juxtaposed DNA ends. This mechanism does not have quality control on the basis of sequence homology and, hence, is not error-free, and can lead to deletions and inversions. Another pathway for DSB repair known as homologous recombination also occurs in somatic cell types, and is much more error-free (Fig. 2) (Kanaar et al., 1998).

Meiotic recombination involves pairing of homologous chromosomes, followed by the exchange of genetic information between non-sister chromatids. The resulting cross-overs are essential for correct segregation of the chromosomes, and play an important role in creating genetic diversity among individuals within a population. Meiotic recombination requires endogenously induced breaking and subsequent ligation of DNA molecules. Therefore, it is not surprising that proteins that mediate recombination between sister chromatids during DSB repair in somatic cells also function during meiotic recombination in germ cells. Evidently, it is not desirable that meiotic DSBs, associated with the process of crossing-over, are repaired by an error-prone pathway. Moreover, meiotic recombination would be seriously hindered if the induced DSBs in meiotic cells were recognized by proteins of the end-joining pathway. Indeed, the expression of Ku70, which is involved in this non-homologous end-joining process, is suppressed in early prophase spermatocytes (Goedecke et al., 1999).

The group of genes encoding proteins involved in the homologous recombination pathway for error-free repair of DSBs, as identified in yeast, is called the RAD52 epistasis group (consisting of: RAD50, RAD51, RAD52, RAD54, RAD55, RAD57, RAD59, MRE11 and XRS2) (Shinohara and Ogawa, 1999).

There are at least two major differences between homologous recombination repair in somatic cells and DNA breaking and repair during gametogenic meiotic recombination. First, DSBs in somatic cells result from damage imposed on the DNA, for example, by environmental factors such as irradiation. However, during the meiotic prophase, the DSBs are induced in a controlled manner, by the action of a specific topoisomerase II variant, SPO11. This protein is conserved from yeast to humans, and its expression is strongly induced in meiotic cells (Bergerat et al., 1997; Keeney et al., 1997, 1999; Romanienko and Camerini-Otero, 1999). Whereas DSBs in somatic cells will occur at random sites, the number and location of induced meiotic DSBs appear to be quite well controlled. The cross-over sites can be identified by immunostaining of foci containing the mismatch repair protein MLH1. In a typical mouse spermatocyte, there are approximately 23 cross-overs. The number of cross-overs is somewhat higher in mouse oocytes, in which about 31 sites have been reported (Baker et al., 1996). In human spermatocytes, an even greater number of MLH1 foci are detected (Fig. 3). This analysis allows for studies on crossingover frequency in spermatocytes isolated from human testis biopsies.

The second major differences between homologous recombination repair in somatic cells and DNA breaking and repair during gametogenic meiotic recombination is that, in somatic cells, recombinational repair of DSBs involves sister chromatids, whereas meiotic recombination requires homologous pairing between non-sister chromatids. Several meiosis-specific aspects of homologous recombination probably are mediated by meiosis-specific variants of proteins acting during homologous recombination DNA repair in somatic cells, for example, DMC1, a meiosisspecific RecA-like protein. There are at least seven homologues of Escherichia coli RecA (or RAD51 in yeast) in mammals (RAD51/51B/51C/51D, XRCC2/3, DMC1) (Shinohara and Ogawa, 1999). RecA/RAD51 is a singlestrand-DNA binding protein that has DNA-dependent ATPase activity and stimulates strand exchange activities. DMC1 is essential for meiosis in yeast and mice (Pittman et al., 1998a; Shinohara and Ogawa, 1999), and may have a distinct role in promoting exchange between the homologous chromosomes, rather than between sister chromatids. However, the true RAD51 orthologue and several other RAD51 homologues are also highly expressed in the testis (Shinohara et al., 1993; Cartwright et al., 1998; Dosanjh et al., 1998; Pittman et al., 1998b), and may participate in meiotic recombination. Immunocytochemical analysis of DMC1 and RAD51 in meiotic cells shows colocalization of both proteins on the synaptonemal complexes of zygotene-pachytene spermatocytes (Tarsounas et al., 1999). The functional relevance of 
meiotic expression of RAD51 in mammals is difficult to determine, owing to the early embryonic lethality of homozygous mutant mice (Thacker, 1999). Lethal phenotypes of knockout mice also hamper studies on the meiotic functions of the mammalian homologues of yeast RAD50 and MRE11 (Haber, 1998; Luo et al., 1999). In yeast, these proteins form a complex together with a protein called XRS2. This complex plays an important role in the repair of double strand breaks through end-joining, but it is also involved in homologous recombination (Haber, 1998). In addition, in collaboration with SPO11, RAD50-MRE11XRS2 is required for the induction of DSBs in meiosis in yeast (Haber, 1998). In mammals, there is a similar complex of RAD50-MRE11, which also includes a protein named nibrin (Petrini, 1999). The genes encoding these three proteins are highly expressed in the testis, and RAD50 and MRE11 colocalize at discrete sites along the synaptonemal complexes in mouse spermatocytes (Goedecke et al., 1999).

In yeast, RAD52 functions as a cofactor in the strandexchange reaction mediated by RAD51, and RAD54 belongs to the large SNF2/SWI2 family of DNA-dependent ATPases (Kanaar et al., 1998). These proteins are essential for meiosis in yeast. In mice, mutagenic inactivation of RAD52 (Rijkers et al., 1998) and RAD54 (Essers et al., 1997 ) results in a viable and quite normal phenotype, in contrast to the lethal phenotype of RAD50 and RAD51 knockout mice, giving hope that it may be possible to analyse a specific gametogenic phenotype. However, in the RAD52 and RAD54 knockout mice, gametogenesis does not appear to be impaired but an explanation for this can be found in the functional redundancy of RAD52 and RAD54 with other proteins that exert a similar function. For example, a human $R A D 54 B$ gene has been identified, that is expressed in multiple tissues, but in large amounts in the testis (Hiramoto et al., 1999). Taken together, it appears that meiotic recombination requires components of the machinery used by somatic cells to repair accidental double strand breaks.

\section{Post-replication repair and spermatogenesis}

The third DNA repair pathway discussed is very complicated. The post-replication repair pathway does not exert DNA repair activity, but rather allows mitotic somatic cells in $\mathrm{S}$ phase to proceed with DNA replication over a DNA template containing damaged bases, in an error-free or error-prone manner (Fig. 2). This mechanism is important for rescuing the cells from premature termination of DNA replication, which would otherwise lead to unscheduled cell death. Evidently, this mechanism relies on other DNA repair pathways to repair the DNA damage after completion of DNA replication. Several proteins of the post-replication repair pathways play an important and intriguing role in gametogenesis, in particular during the meiotic prophase and in the post-meiotic development of spermatids. One of the many questions with regard to the role of proteins involved in post-replication repair in gametogenesis is whether this role concerns the post-replication repair process itself or other aspects of chromatin dynamics.

In the yeast Saccharomyces cerevisiae, genes involved in post-replication DNA repair are members of the RAD6 epistasis group. The expression of the yeast RAD6 gene is strongly induced by UV exposure and during meiosis, and null mutants show a very pleiotrophic phenotype, including defects in gene silencing, and impaired sporulation (Lawrence, 1994). In mammalian cells, two highly conserved homologues of this gene have been identified: the $X$ chromosomal HR6A and the autosomal HR6B genes (Koken et al., 1991). In view of the high level of evolutionary conservation, and the induction of RAD6 expression during meiosis and its role in sporulation, it is possible that the HR6A and HR6B proteins are involved in mammalian gametogenesis. However, it is important to note that the genes encoding these two proteins are expressed in virtually all cell types. When HR6A and $H R 6 B$ knockout mice were generated, no major phenotype was observed for somatic tissues, which is explained by the functional redundancy of the proteins encoded by these two genes (the proteins show 96\% amino acid sequence identity) (Roest et al., 1996, H. P. Roest, unpublished). Together, these proteins also play an important role in mammals, because HR6A-HR6B double knockout animals are not obtained; this condition results in very early embryonic death (H. P. Roest, unpublished). With regard to a role for the mammalian RAD6 homologues in gametogenesis, the results exceeded expectations. The HR6A knockout mice showed a maternal-effect infertility (block of embryonic development at the two-cell stage), with normal male fertility (Grootegoed et al., 1998; H. P. Roest, unpublished). In contrast, HR6B knockout mice demonstrated impaired spermatogenesis and malerestricted infertility (Roest et al., 1996).

The RAD6 gene encodes a ubiquitin-conjugating enzyme (E2) which functions in the ubiquitin pathway to modify and breakdown proteins. The role of the ubiquitin pathway in gametogenesis is discussed in detail elsewhere (Baarends et al., 1999a) but the following may serve as a summary of several important points. Protein targets for the RAD6-dependent ubiquitination probably include histones $\mathrm{H} 2 \mathrm{~A}$ and $\mathrm{H} 2 \mathrm{~B}$, and possibly other chromosomal proteins (Baarends et al., 1999b; Robzyk et al., 2000). In mammalian spermatogenesis, HR6B might play some role in the histone-to-protamine transition that takes place in condensing spermatids (Baarends et al., 1999b). In addition, HR6B-dependent ubiquitination may target proteins involved in the control of gene expression and chromatin dynamics during the meiotic prophase. The HR6B knockout males show increased apoptosis of pachytene spermatocytes, but there is no meiotic arrest (Baarends et al., 1999a; W. M. Baarends, unpublished). The major loss of germ cells occurs during post-meiotic development, and only a small number of highly abnormal spermatozoa are found in the epididymis (Roest et al., 1996). 
The yeast $R A D 18$ gene encodes a protein with a RINGzinc-finger domain and a classical DNA binding zincfinger. Both domains are highly conserved among RAD18, two fungal homologues (NuvA and Uvs-2), and the cloned mammalian homologues mRAD18Sc (van der Laan et al., in press), and hRAD18 (Tateishi et al., 2000; Xin et al., 2000). The RING-zinc-finger domain is present in proteins involved in multiprotein complexes, indicating that the domain mediates protein-protein interactions. In particular, the RING-zinc-finger domain is present in proteins with ubiquitin-ligating enzyme activity (E3 enzymes), which are involved in the control of the substrate specificity of the ubiquitin-conjugating (E2) enzymes (Lorick et al., 1999). The yeast RAD6 protein forms a stable complex with RAD18, and this complex possesses ubiquitin-conjugating and DNA-binding activities (Bailly et al., 1994). hRAD18 has also been shown to interact with HR6A and HR6B (Tateishi et al., 2000; Xin et al., 2000).

The mouse RAD18 homologue $m R A D 18 S c$ shows the highest mRNA expression in testis, in particular in primary spermatocytes (van der Laan et al., in press). Hence, during mammalian spermatogenesis, mRAD18Sc (acting as an E3 enzyme) may be involved in aspects of the function of HR6B (acting as an E2 enzyme) in the ubiquitin pathway. The protein targets of HR6B in germ cells, possibly complexed with mRAD18Sc, need to be identified. These targets may be involved in general aspects of gametogenic chromatin structure dynamics and gene expression, rather than in postreplication DNA repair.

The post-replication DNA repair pathway becomes active when the DNA replication machinery is stalled at a lesion. Specialized polymerases take over, to add just a few nucleotides to the growing DNA chain opposite the lesion, over the damaged template. Subsequently, the DNA replication machinery reinitiates DNA replication. Many new findings with regard to these specialized polymerases have been published for both prokaryotic and eukaryotic cells (Friedberg and Gerlach, 1999). One of the many DNA polymerases in $S$. cerevisiae is encoded by $R A D 30$. In humans, the homologue RAD30A is mutated in patients with the xeroderma pigmentosum variant, resulting in increased incidence of sunlight-induced skin cancers (Masutani et al., 1999). The expression profile of the RAD30A gene has not been determined, but the second mammalian $R A D 30$ homologue $(R A D 30 B)$ is expressed predominantly in human testis. In situ hybridization for $R A D 3 O B$ in mouse testis revealed that the gene is mainly expressed in round spermatids (McDonald et al., 1999). The functional relevance of such a late expression, after completion of the major DNA replication and recombination events, can be questioned. However, it is possible that RAD30B functions in spermatids to repair lesions induced by exogenous factors (McDonald et al., 1999). Alternatively, use of the post-replication DNA repair pathway during gametogenesis may facilitate mutagenesis. In bacteria, translesion DNA synthesis may generate a background of mutations upon which selection could operate in response to changes in environmental conditions (Echols, 1981; Friedberg and Gerlach, 1999). There might be such a role for the mutagenic activity of the post-replication DNA repair pathway in mammalian gametogenesis, to generate genomic diversity among spermatozoa. This idea is highly speculative, and thus far supported only by observations of high testicular expression of several mutagenesis-related polymerases.

The results of future experiments may show whether the mutagenic DNA polymerases, which are involved in translesion replication during postreplication DNA repair, play some specific role in gametogenesis. As for many other proteins involved in pathways that are also important for normal somatic cell development and function, generation of straightforward gene knockout mouse models will probably not be adequate. Rather, it is likely to be necessary to inactivate the respective gene specifically during gametogenesis, using a more complicated conditional gene knockout strategy.

\section{DNA damage-induced cell cycle checkpoint genes}

In spite of the control exerted by DNA repair pathways, gametogenic cells may encounter DNA damage to such an extent that further development of these cells is prevented. In somatic mitotically active cells, a control mechanism that checks the integrity of the genome operates at different points during the cell cycle. This mechanism is capable of blocking the cell cycle until the DNA damage has been repaired, thus ensuring faithful transmission of DNA to daughter cells. If DNA damage cannot be repaired owing to quantitative or qualitative failure of DNA repair mechanisms, the activity of cell cycle checkpoint genes may result in cell death through apoptosis. During gametogenesis, the activity of a meiotic cell cycle checkpoint is illustrated by the pachytene arrest observed in several mouse models for male infertility. Pachytene arrest is also a relatively frequent observation in testis biopsies taken from infertile human males.

A large number of proteins is involved in the regulation of DNA damage-induced cell cycle control. The activities of only a limited number of genes involved in this mechanism are discussed here.

The human genetic disorder ataxia telangiectasia (A-T), caused by mutation in the ATM gene, is characterized by radiosensitivity, defective cell cycle checkpoint activation, chromosomal instability and infertility (Meyn, 1999). ATM is a member of a family of protein kinases with similarity to phosphatidylinositol-3 kinases. ATM knockout mice are infertile owing to a meiotic arrest at the zygotenepachytene stage (Xu et al., 1996). Aberrant telomere clustering and chromosome fragmentation are observed (Barlow et al., 1998). ATM is expressed in oocytes and spermatocytes and is thought to monitor meiosis through a role in the control or surveillance of meiotic progression, similar to its function in mitotic cell cycle progression. Keegan et al. (1996) suggested that ATM localizes to the 
paired axes of the synaptonemal complexes in zygotene and pachytene spermatocytes. However, this finding was not confirmed by Moens et al. (1999). ATR, which is an ATM-like protein, localizes to the unpaired axes of meiotic prophase chromosomes (Keegan et al., 1996; Moens et al., 1999). Mutation of ATR results in an embryonic lethal phenotype (Brown and Baltimore, 2000). ATR and ATM most likely exert their function through phosphorylation of proteins associated with complexes that localize to damaged DNA. An interesting substrate of ATM is BRCA1 (Cortez et al., 1999). This protein, and the functionally related BRCA2, are encoded by breast cancer susceptibility genes, and seem to be involved in several DNA repair mechanisms: nucleotide excision repair, homologous recombination repair, and double-strand break repair (Welcsh et al., 2000). BRCA1 and BRCA2 are both very large, but otherwise completely different proteins. BRCA1 may function as a ubiquitin ligase (E3 enzyme) through its RING finger domain. Both proteins contain sequences that may perform transcription activation functions. In addition, both proteins are capable of interacting with RAD51, and colocalization with RAD51 has been reported in meiotic cells (Scully et al., 1997). BRCA1 knockout and $B R C A 2$ knockout mice show very severe phenotypes, and most animals die during embryonic development (Welcsh et al., 2000). However, a particular truncating BRCA2 mutation allows survival of some homozygous mutant mice to adulthood (Connor et al., 1997). These mice show male and female infertility. In males, spermatogenesis is affected even before meiosis has started, and testicular tubules from adults contain only Sertoli cells (Connor et al., 1997). For BRCA1, it has been shown that BRCA1P53 double knockout mice, in which the P53 mutation partially rescues the embryonic lethal phenotype, show male-restricted infertility, owing to meiotic failure (Cressman et al., 1999).

\section{Concluding remarks}

A vast number of genes encoding proteins that take part in different DNA repair mechanisms show enhanced or specialized expression during gametogenesis. Indeed, many knockout mouse models in which one of these genes is inactivated by targeted mutation show male or female infertility, or both. In future studies, it will be important to establish what the precise relationship is between DNA repair and mutagenesis, and other aspects of gametogenesis related to DNA and chromatin dynamics, such as maintenance of telomere length, genomic imprinting, and genomic instability. With respect to genomic instability, there are unexplained observations on the expansion or contraction of DNA trinucleotide repeats, which are associated with several human neurodegenerative diseases (Richards and Sutherland, 1997), and for which there is a striking difference between somatic and germline cells. It is not known which fundamental differences between somatic and germ cells underlie this phenomenon, but differential activities of DNA repair mechanisms may be involved.

Post-meiotic spermatids have completed all major DNA replication and recombination events. The capacity to repair DNA damage becomes restricted in elongating spermatids (van Loon et al., 1993), when the condensing chromatin (histone-to-protamine transition) limits the proteins of the DNA repair machinery gaining access to the DNA. However, before the point of chromatin silencing is reached, DNA repair mechanisms probably continue to play a quite prominent role in several aspects of chromatin dynamics in round and elongating spermatids.

Finally, when DNA damage may have escaped gametogenic DNA repair, or when DNA damage occurs in spermatozoa, to a certain extent such damage is successfully repaired during the process of fertilization (Brandriff and Pedersen, 1981; Ashwood-Smith and Edwards, 1996), which includes the formation of pronuclei, DNA replication, and pronuclear fusion, before formation of the zygote.

The authors would like to thank Henk P. Roest and Jan H. J. Hoeijmakers for stimulating discussions and invaluable support. Furthermore, they are grateful to Evelyne Wassenaar for expert technical assistance leading to Fig. 3. The antibody targeting SCP3 was kindly provided by Christa Heyting. This work was supported by the Dutch Science Foundation (NWO) through GB-MW (Medical Sciences) and by the Dutch Cancer Society (EUR 99-2003).

\section{References}

Key references are identified by asterisks.

Agulnik AI, Bishop CE, Lerner JL, Agulnik SI and Solovyev VV (1997) Analysis of mutation rates in the SMCY/SMCX genes shows that mammalian evolution is male driven Mammalian Genome 8 134-138

*Arnheim N and Shibata D (1997) DNA mismatch repair in mammals: role in disease and meiosis Current Opinion in Genetics and Development 7 364-370

Ashwood-Smith MJ and Edwards RG (1996) DNA repair by oocytes Molecular Human Reproduction 2 46-51

Baarends WM, Roest HP and Grootegoed JA (1999a) The ubiquitin system in gametogenesis Molecular and Cellular Endocrinology 151 5-16

*Baarends WM, Hoogerbrugge JW, Roest HP, Ooms M, Vreeburg J, Hoeijmakers JHJ and Grootegoed JA (1999b) Histone ubiquitination and chromatin remodeling in mouse spermatogenesis Developmental Biology 207 322-333

Bailly V, Lamb J, Sung P, Prakash S and Prakash L (1994) Specific complex formation between yeast RAD6 and RAD18 proteins: a potential mechanism for targeting RAD6 ubiquitin-conjugating activity to DNA damage sites Genes and Development 8 811-820

Baker SM, Bonner CE, Zhang L, Plug AW, Robatzek M, Warren G, Elliott EA, Yu J, Ashley T, Arnheim N, Flavell RA and Liskay RM (1995) Male mice defective in the DNA mismatch repair gene PMS2 exhibit abnormal chromosome synapsis in meiosis Cell 82 309-319

Baker SM, Plug AW, Prolla TA, Bronner CE, Harris AC, Yao X, Christie DM, Monell C, Arnheim N, Bradley A, Ashley T and Liskay RM (1996) Involvement of mouse Mlh1 in DNA mismatch repair and meiotic crossing over Nature Genetics 13 336-342

Barlow C, Liyanage M, Moens PB, Tarsounas M, Nagashima K, Brown K, Rottinghaus S, Jackson SP, Tagle D, Ried T and Wynshaw-Boris A (1998) Atm deficiency results in severe meiotic disruption as early as leptonema of prophase I Development 125 4007-4017

Bergerat A, de Massy B, Gadelle D, Varoutas PC, Nicolas A and Forterre 
P (1997) An atypical topoisomerase II from Archaea with implications for meiotic recombination Nature 386 414-417

Brandriff B and Pedersen RA (1981) Repair of the ultraviolet-irradiated male genome in fertilized mouse eggs Science 211 1431-1433

Brown EJ and Baltimore D (2000) ATR disruption leads to chromosomal fragmentation and early embryonic lethality Genes and Development 14 397-402

Cartwright R, Dunn AM, Simpson PJ, Tambini CE and Thacker J (1998) Isolation of novel human and mouse genes of the recA/RAD51 recombination-repair gene family Nucleic Acids Research 26 1653-1659

Connor F, Bertwistle D, Mee PJ, Ross GM, Swift S, Grigorieva E, Tybulewicz VL and Ashworth A (1997) Tumorigenesis and a DNA repair defect in mice with a truncating Brca2 mutation Nature Genetics 17 423-430

Cortez D, Wang Y, Qin J and Elledge SJ (1999) Requirement of ATMdependent phosphorylation of Brca1 in the DNA damage response to double-strand breaks Science 286 1162-1166

Cressman VL, Backlund DC, Avrutskaya AV, Leadon SA, Godfrey V and Koller BH (1999) Growth retardation, DNA repair defects, and lack of spermatogenesis in BRCA1-deficient mice Molecular and Cellular Biology $197061-7075$

de Vries SS, Baart EB, Dekker M, Siezen A, de Rooij DG, de Boer P and te Riele H (1999) Mouse MutS-like protein Msh5 is required for proper chromosome synapsis in male and female meiosis Genes and Development 13 523-531

de Wind N, Dekker M, Claij N, Jansen L, van Klink Y, Radman M, Riggins G, van der Valk M, van't Wout K and te Riele H (1999) HNPCC-like cancer predisposition in mice through simultaneous loss of Msh3 and Msh6 mismatch-repair protein functions Nature Genetics 23 359-362

Dosanjh MK, Collins DW, Fan W, Lennon GG, Albala JS, Shen Z and Schild D (1998) Isolation and characterization of RAD51C, a new human member of the RAD51 family of related genes Nucleic Acids Research 26 1179-1184

Echols H (1981) SOS functions, cancer and inducible evolution Cell 25 $1-2$

Edelmann W, Cohen P, Kane M, Lau K, Morrow B, Bennett S, Umar A Kunkel T, Cattoretti G, Chaganti R, Pollard JW, Kolodner RD and Kucherlapati R (1996) Meiotic pachytene arrest in MLH1-deficient mice Cell 85 1125-1134

Edelmann W, Cohen PE, Kneitz B, Winand N, Lia M, Heyer J, Kolodner R, Pollard JW and Kucherlapati R (1999) Mammalian MutS homologue 5 is required for chromosome pairing in meiosis Nature Genetics 21 $123-127$

Essers J, Hendriks RW, Swagemakers SMA, Troelstra C, Wit Jd, Bootsma D, Hoeijmakers JHJ and Kanaar R (1997) Disruption of mouse RAD54 reduces ionizing radiation resistance and homologous recombination Cell 89 195-204

*Friedberg EC and Gerlach VL (1999) Novel DNA polymerases offer clues to the molecular basis of mutagenesis Cell 98 413-416

Goedecke W, Eijpe $\mathrm{M}$, Offenberg $\mathbf{H H}$, van Aalderen $\mathbf{M}$ and Heyting $\mathrm{C}$ (1999) Mre11 and Ku70 interact in somatic cells, but are differentially expressed in early meiosis Nature Genetics 23 194-198

Grootegoed JA, Baarends WM, Roest HP and Hoeijmakers JH (1998) Knockout mouse models and gametogenic failure Molecular and Cellular Endocrinology 145 161-166

Haber JE (1998) The many interfaces of Mre11 Cell 95 583-586

Hiramoto T, Nakanishi T, Sumiyoshi T et al. (1999) Mutations of a novel human RAD54 homologue, RAD54B, in primary cancer Oncogene 18 3422-3426

*Hurst LD and Ellegren $\mathbf{H}$ (1998) Sex biases in the mutation rate Trends in Genetics 14 446-452

Kanaar R, Hoeijmakers JH and van Gent DC (1998) Molecular mechanisms of DNA double strand break repair Trends in Cell Biology 8 483-489

Keegan KS, Holtzman Da, Plug AW, Christenson ER, Brainerd EE, Flaggs G, Bentley NJ, Taylor EM, Meyn MS, Moss SB et al. (1996) The Atr and Atm protein kinases associate with different sites along meiotically pairing chromosomes Genes and Development 10 2383-2388

Keeney S, Giroux CN and Kleckner N (1997) Meiosis-specific DNA double-strand breaks are catalyzed by Spo11, a member of a widely conserved protein family Cell 88 375-384

Keeney S, Baudat F, Angeles M, Zhou ZH, Copeland NG, Jenkins NA Manova K and Jasin M (1999) A mouse homolog of the Saccharomyces cerevisiae meiotic recombination DNA transesterase Spo11p Genomics 61 170-182

Kneitz B, Cohen PE, Avdievich E, Zhu L, Kane MF, Hou H, Jr, Kolodner RD, Kucherlapati R, Pollard JW and Edelmann W (2000) MutS homolog 4 localization to meiotic chromosomes is required for chromosome pairing during meiosis in male and female mice Genes and Development 14 1085-1097

Koken MH, Reynolds P, Jaspers-Dekker I, Prakash L, Prakash S, Bootsma D and Hoeijmakers JH (1991) Structural and functional conservation of two human homologs of the yeast DNA repair gene RAD6 Proceedings National Academy of Sciences USA 88 8865-8869

Lawrence C (1994) The RAD6 repair pathway in Saccharomyces cerevisiae: what does it do, and how does it do it? BioAssays 16 253-258

Lipkin SM, Wang V, Jacoby R, Banerjee-Basu S, Baxevanis AD, Lynch HT, Elliott RM and Collins FS (2000) MLH3: a DNA mismatch repair gene associated with mammalian microsatellite instability Nature Genetics 24 27-35

Lorick KL, Jensen JP, Fang S, Ong AM, Hatakeyama S and Weissman AM (1999) RING fingers mediate ubiquitin-conjugating enzyme (E2)dependent ubiquitination Proceedings National Academy of Sciences USA $9611364-11369$

Luo G, Yao MS, Bender CF, Mills M, Bladl AR, Bradley A and Petrini JH (1999) Disruption of mRad50 causes embryonic stem cell lethality, abnormal embryonic development, and sensitivity to ionizing radiation Proceedings National Academy of Sciences USA 96 7376-7381

McDonald JP, Rapic-Otrin V, Epstein JA, Broughton BC, Wang X, Lehmann AR, Wolgemuth DJ and Woodgate R (1999) Novel human and mouse homologs of Saccharomyces cerevisiae DNA polymerase eta Genomics 60 20-30

Masutani C, Kusumoto R, Yamada A, Dohmae N, Yokoi M, Yuasa M, Araki M, Iwai S, Takio K and Hanaoka F (1999) The XPV (xeroderma pigmentosum variant) gene encodes human DNA polymerase eta Nature 399 700-704

Meyn MS (1999) Ataxia-telangiectasia, cancer and the pathobiology of the ATM gene Clinical Genetics 55 289-304

Moens PB, Tarsounas M, Morita T, Habu T, Rottinghaus ST, Freire R, Jackson SP, Barlow C and Wynshaw-Boris A (1999) The association of ATR protein with mouse meiotic chromosome cores Chromosoma 108 95-102

Paquis-Flucklinger V, Santucci-Darmanin S, Paul R, Saunieres A, TurcCarel C and Desnuelle C (1997) Cloning and expression analysis of a meiosis-specific MutS homolog: the human MSH4 gene Genomics 44 188-194

Petrini JH (1999) The mammalian Mre11-Rad50-nbs1 protein complex: integration of functions in the cellular DNA-damage response American Journal of Human Genetics 64 1264-1269

Pittman DL, Cobb J, Schimenti KJ, Wilson LA, Cooper DM, Brignull E, Handel MA and Schimenti JC (1998a) Meiotic prophase arrest with failure of chromosome synapsis in mice deficient for Dmc1, a germlinespecific RecA homolog Molecular Cell 1 697-705

Pittman DL, Weinberg LR and Schimenti JC (1998b) Identification, characterization, and genetic mapping of Rad51d, a new mouse and human RAD51/RecA-related gene Genomics 49 103-111

Richards RI and Sutherland GR (1997) Dynamic mutation: possible mechanisms and significance in human disease Trends in Biochemical Sciences 22 432-436

Rijkers T, Van Den Ouweland J, Morolli B, Rolink AG, Baarends WM Van Sloun PP, Lohman PH and Pastink A (1998) Targeted inactivation of mouse RAD52 reduces homologous recombination but not resistance to ionizing radiation Molecular and Cellular Biology $\mathbf{1 8}$ 6423-6429

Robzyk K, Recht J and Osley MA (2000) Rad6-dependent ubiquitination of histone H2B in yeast Science 287 501-504

*Roest HP, Klaveren van J, Wit de J et al. (1996) Inactivation of the HR6B ubiquitin-conjugating DNA repair enzyme in mice causes a defect in 
spermatogenesis associated with chromatin modification Cell 86 799-810

Romanienko PJ and Camerini-Otero RD (1999) Cloning, characterization, and localization of mouse and human SPO11 Genomics 61 156-169

Santucci-Darmanin S, Walpita D, Lespinasse F, Desnuelle C, Ashley T and Paquis-Flucklinger V (2000) MSH4 acts in conjunction with MLH1 during mammalian meiosis FASEB Journal 14 1539-1547

Scully R, Chen J, Plug AW, Xiao Y, Weaver D, Feunteun J, Ashley T and Livingston DM (1997) Association of BRCA1 with Rad51 in mitotic and meiotic cells Cell 88 265-275

Shinohara A and Ogawa T (1999) Rad51/RecA protein families and the associated proteins in eukaryotes Mutation Research 435 13-21

Shinohara A, Ogawa H, Matsuda Y, Ushio N, Ikeo K and Ogawa T (1993) Cloning of human, mouse and fission yeast recombination genes homologous to RAD51 and recA Nature Genetics 4 239-243

Tarsounas M, Morita T, Pearlman RE and Moens PB (1999) RAD51 and DMC1 form mixed complexes associated with mouse meiotic chromosome cores and synaptonemal complexes Journal of Cell Biology 147 207-220

Tateishi S, Sakuraba Y, Masuyama S, Inoue H and Yamaizumi M (2000) Dysfunction of human Rad18 results in defective postreplication repair and hypersensitivity to multiple mutagens Proceedings National Academy of Sciences USA 97 7927-7932

Thacker J (1999) A surfeit of RAD51-like genes? Trends in Genetics 15 166-168 van der Laan R, Roest HP, Hoogerbrugge JW, Smit EM, Slater R, Baarends WM, Hoeijmakers JH and Grootegoed JP (2000) Characterization of mRAD18Sc, a mouse homolog of the yeast postreplication repair gene RAD18 Genomics 69 86-94

van Loon AA, Sonneveld E, Hoogerbrugge J, van der Schans GP, Grootegoed JA, Lohman PH and Baan RA (1993) Induction and repair of DNA single-strand breaks and DNA base damage at different cellular stages of spermatogenesis of the hamster upon in vitro exposure to ionizing radiation Mutation Research 294 139-148

Wang TF, Kleckner N and Hunter N (1999) Functional specificity of MutL homologs in yeast: evidence for three Mlh1-based heterocomplexes with distinct roles during meiosis in recombination and mismatch correction Proceedings National Academy of Sciences USA 9613 914-13919

*Welcsh PL, Owens KN and King MC (2000) Insights into the functions of BRCA1 and BRCA2 Trends in Genetics 16 69-74

Winand NJ, Panzer JA and Kolodner RD (1998) Cloning and characterization of the human and Caenorhabditis elegans homologs of the Saccharomyces cerevisiae MSH5 gene Genomics 53 69-80

Xin H, Lin W, Sumanasekera W, Zhang Y, Wu X and Wang Z (2000) The human RAD18 gene product interacts with HHR6A and HHR6B Nucleic Acids Research 28 2847-2854

Xu Y, Ashley T, Brainerd EE, Bronson RT, Meyn MS and Baltimore D (1996) Targeted disruption of ATM leads to growth retardation, chromosomal fragmentation during meiosis, immune defects, and thymic lymphoma Genes and Development 10 2411-2422 